\title{
Knots, Slipknots, and Ephemeral Knots in Random Walks and Equilateral Polygons
}

\author{
Kenneth C. Millett*
}

December 1, 2008

\begin{abstract}
The probability that a random walk or polygon in the 3-space or in the simple cubic lattice contains a knot goes to one at the length goes to infinity. Here, we prove that this is also true for slipknots consisting of unknotted portions, called the slipknot, that contain a smaller knotted portion, called the ephemeral knot. As is the case with knots, we prove that any topological knot type occurs as the ephemeral knotted portion of a slipknot.
\end{abstract}

\section{Introduction}

Self-avoiding random walks and polygons in 3-space or in the cubic lattice provide a popular model for linear polymers under certain physical conditions. With increasing length, the probability that a random walk or polygon contains a knot goes to one [SW88, Pip89, DPS94, Dia95] proving a conjecture of Frisch and Wasserman [FW61] and of Delbruck [Del62]. In addition, this knotted portion can be of any desired topological type. As a consequence, the influence of knotting in the statistical mechanical and physical properties of polymers [Edw67, VKK05], their occurrence in DNA [WC86] and in proteins [Man94, Tay00, VMK06], and their impact upon the scaling of of physical properties of macromolecules [OTJvRW98a, Deu99, Gro00, $\mathrm{DDM}^{+}$03] have inspired wide ranging research. In this paper, we extend the knotting concept to encompass a more fragile knotted structure that can be found in random walks and polygons and which has recently been found in natural biological structures. An ephemeral knot in a random walk or polygon is a knotted portion that is contained in a larger unknotted portion which we will call a slipknot. Slipknots have been identified in proteins [KYY07]. We conjecture that they are also present in a wide range of other macromolecular structures. They appear, however, to have avoided experimental or theoretical detection. For polymers in a good solvent, i.e. in the $\theta$ condition, one expects that knots are quite localized

${ }^{*}$ Department of Mathematics, University of California, Santa Barbara, Santa Barbara, CA 93106, USA, millett@math.ucsb.edu 
although it has been quite challenging to acquire fully robust measures of the relative scale [OTJvRW98b, $\mathrm{KOV}^{+} 00$, MOSZ05, MOSZ07]. The knotted portions of the random walk or polygonal chain have an impact upon the spatial conformation of the chain and, as a consequence, their presence correlates with the physical properties of the modeled polymers. We propose that the presence of slipknots should have an analogous influence upon the associated physical properties of polymers.

We first describe knotting in 3-space and in the cubic lattice. Next, we review several approaches to the identification and quantification of knotting in open chains and describe the properties that recommend the MDS procedure [MDS05, MS05]. Using these criteria, we then show how to extend the methods for knots to show that the probability that a random walk or polygon contains a slipknot knot, and its companion ephemeral knot, goes to one as the length goes to infinity.

\section{Knots, Ephemeral Knots, and Slipknots}

A closed polygon in 3-space is knotted if there is no ambient deformation of Euclidean space taking the polygon to the standard planar circle. While the search for computationally efficient and effective methods to determine the specific structure of knotting for polygons in 3-space is a continuing challenge for topology, the search for an appropriate formulation of knotting of open polygons is even more difficult. From a classical topological point of view, knotting of open polygons is not possible because, if edge lengths are allowed to vary, each open polygon is ambient isotopic to a standard interval in the " $\mathrm{x}$ "-axis in 3 -space (This argument is popularly called the "light-bulb" theorem) and, as a consequence, open polygons are topologically unknotted. They may, however, be geometrically knotted when the edge lengths are fixed. This is confirmed by the examples of Canteralla-Johnson and others [CJ98, Tou01, AET04]. It is unknown, if one considers equilateral polygons, whether or not there are configurations that cannot be deformed to a straight segment preserving the edge lengths. The analogous problem for closed equilateral polygons is also unknown, i.e. "Are there topologically unknotted equilateral polygon configurations in 3space that cannot be deformed to the standard planar equilateral polygon?" These two questions give a sense of the degree of difficulty in describing the knot theory of equilateral polygons in 3-space beyond those having 8 or fewer edges. For these fundamental cases, one knows more about the nature and structure on knotting [CM98, Cal98, Cal01a, Cal01b].

For open polygons, the search of an effective notion of knotting is a matter of continuing effort. In 1994, Mansfield [Man94] proposed the "reasonable-person" approach to the knotting of curves or polygonal arcs in discussing knots in proteins, specifically: "A polygonal arc is knotted if any 'reasonable-person' would say it was knotted." In 2000, Taylor[Tay00] turned this approach into an effective research method with an algorithm to simplify the polygonal arc while holding its endpoints fixed. The objective of this strategy is to make it easier for 
a "reasonable-person" to be able to decide whether or not a knot was present in the arc and to identify its topological knot type. There is, however, an intrinsic uncertainty inherent to the Taylor and analogous algorithms as the resulting terminal configuration is dependent upon specific choices made in the course of the simplification algorithm. Examples [MDS05] show that one can reach different conclusions depending upon the specific sequence of operations employed in implementations of the Taylor algorithm, see Figure 1. Nevertheless, this strategy continues to be the foundation of efforts to identify knotted structure as it is argued that such problematic conformations as statistically rare, e.g. [KYY07, YNK08].

Another strategy that is commonly employed is to convert a polygonal configuration into a closed circular configuration in some specified manner, [JvRSWW92, MOSZ05, VKK05, VMK06, MOSZ07] and assign the knot type of the closed configuration to the open one from which it originates. For closed polygonal configurations, the classical Reidemeister moves allow one to simplify the configuration without changing its knot type. A sufficiently simple polygonal configuration can then be analyzed via a wide range of knot invariants such as the Alexander, Jones, HOMFLY, and Kauffman polynomial invariants. Thus, the fundamental question is "How does one close an open polygon to correctly identify its appropriate knot type?" Perhaps the most basic method is to simply connect the ends of the open polygon by an arc. While giving the "right" answer [MDS05] with sufficient frequency to serve as a viable statistical strategy, one might argue that this strategy is not sufficiently reliable for the analysis of individual instances or when sample sizes are small. Another attractive closure method is to randomly extend the terminal segments to "infinity" where they close the polygon. In practice, this means that the rays from the terminal vertices intersect a very large sphere, one containing the polygon under consideration, in two points that are then joined by a great circle arc on the sphere (or are closed in an equivalent manner) [JvRSWW92]. Another strategy is to connect the end vertices to "infinity" with rays originating from the center of mass of the polygon [VKK05, VMK06] and assign to the configuration the topological knot type. Each of these methods suffers from an inherent instability that, at least from a mathematical perspective, causes some measure of concern about the reliance on a single closure whose character may change in fundamental ways under small perturbations of the vertices that define the polygon. Such measures can, thereby, fail to satisfy the "reasonable-person" criterion.

In order to study the knotting of open off-lattice, or 3-space, polygons and being concerned with certain features of existing methods, Millett, Dobay and Stasiak [MDS05, MS05] proposed a statistical method to identify and describe, quantitavely, the nature of the knotting present in an open polygon. This method was evaluated in a study of knotting in random walks and tested against the previously identified knotting present in protein structures. The MDS Method can be described as follows: given an open polygonal arc, consider the probability distribution, or knotting spectrum, arising from connecting both endpoints of the polygon to a point on the sphere "at infinity," as the 


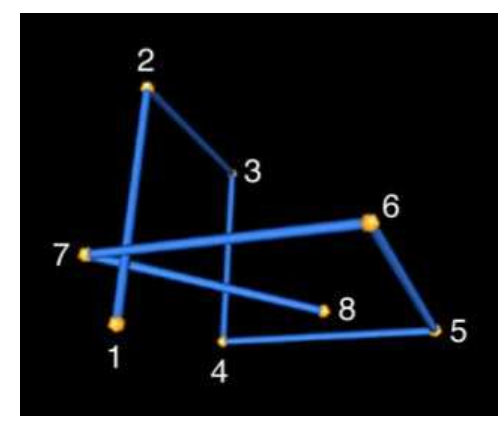

Figure 1: Unknot and Trefoil are possible outcomes [MDS05]

point on the sphere vary with respect to the uniform distribution. For all practical purposes, this spectrum identifies a dominate knot type at the 0.50 level, meaning that a single knot type occurs in more than half the closures. In a test of one thousand 300 step random walks in 3 -space, the 0.50 level test was successful $99.6 \%$ of the time [MDS05]. As a consequence, we conclude that the MDS approach provides a effective research method with which to analyze the knotting of open chains.

We observe that these different definitions of the knotting of open chains, as well as the one employed by Sumners and Whittington [SW88] described next, may be used and will result in the same theorems. In order to set the context for the proofs of our theorems, we review their methods. Considering an open polygon as "frozen in space-time," Sumners-Whittington [SW88] employ a definition that uses a properly embedded ball-knot pair. Precisely, if there is a ball in the simple cubic lattice or in 3-space that properly intersects the polygon (meaning that the boundary sphere of the ball meets the polygon in precisely two points and the interior of the arc defined by them lies in the interior of the ball) such that the ball-arc pair is knotted, one says that the open polygon contains the knot type determined by the ball pair. This definition "makes sense" because one can not eliminate a knot by tying another knot in another, distant, part of the polygon, i.e. knots do not have "inverses." Using this definition of knotting in open chains, they prove that the probability that a random closed self-avoiding random walk or polygon in the simple cubic lattice is knotted goes to one as its length goes to infinity. The key result is an estimate of the growth in the number of self-avoiding open polygons in the simple cubic lattice that contain knotted ball pairs.

\subsection{Knots, Slipknots, and Ephemeral knots in the Simple Cubic Lattice}

As mentioned earlier, the goal of this paper is the extension of the following theorem: 

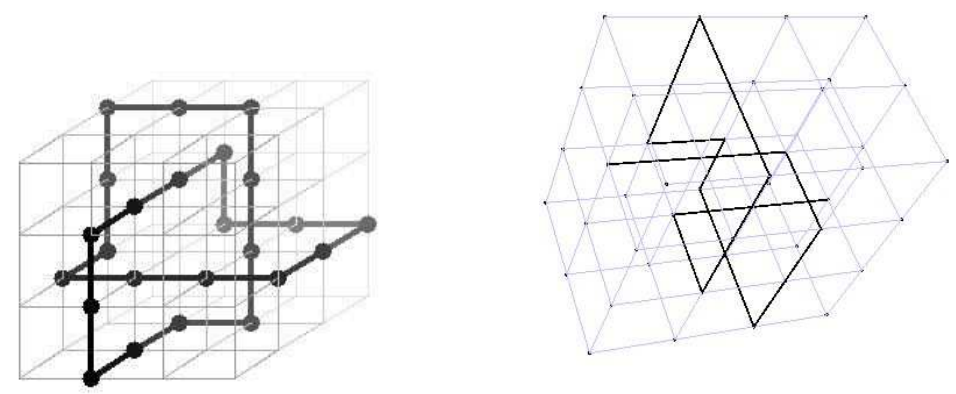

Figure 2: A closed 24 edge trefoil knot and the analogous Sumners-Whittington tight trefoil Kesten pattern

Theorem 2.1. Sumners and Whittington, Pippenger[SW88, Pip89] All except exponentially few sufficiently long self-avoiding polygons on the simple cubic lattice contain a knot of any given topological type.

The proof is based upon the Kesten Pattern Lemma 2.2 [Kes63] and the observation that, for example, Diao's smallest lattice trefoil knot [Dia93], shown in Figure 2, can be modified to provide the "open" trefoil knot also shown in Figure 2 , which has the necessary properties to use the pattern lemma. The relationship between the last two definitions of knotting in open chains is complicated for several reasons. In the Sumners-Whittington definition, the embedding of the 3-ball can be spatially complex and exceedingly difficult to recognize in a numerical study. In addition, if one attempts to restrict the class of 3-balls to only the spherical ones, it is likely that many instances of "reasonable-person" knotting will not be readily recognizable. It is this difficulty, in contrast to its power to support a mathematical proof of knotting, that makes the MDS method an attractive experimental tool despite the difficulty inherent in employing it in the construction of mathematical proofs. Applying the MDS method to the polygonal segment employed by Sumners and Whittington, shown in Figure 2, we find that the knotting spectrum, for a sample of size 10,000, consists of $31.4 \%$ unknots and $68.6 \%$ trefoil knots thereby demonstrating that the configuration is a trefoil knot according to the MDS 50\% standard.

Proof. Key to the proof of the theorem and the Kesten Pattern Lemma is the growth of self-avoiding random walks and polygons. Let $W(n)$ denote the set of self-avoiding random walks in the simple cubic lattice starting at $\{0,0,0\}$ and let $P(n)$ denote the set of self-avoiding polygons in the simple cubic lattice based at $\{0,0,0\}$. Define $w_{n}=\|W(n)\|$ and $p_{n}=\|P(n)\|$. Hammersley and Morton [HM54] have shown that

$$
0<\lim _{n \rightarrow \infty} n^{-1} \log w_{n}=\inf _{n>0} w_{n}=\kappa<\infty .
$$

Hammersley [Ham61] also showed that 


$$
\lim _{n \rightarrow \infty} n^{-1} \log p_{n}=\lim _{n \rightarrow \infty} n^{-1} \log p_{n}=\kappa .
$$

As noted above, the Sumners-Whittington lattice trefoil shown in Figure 2 is an example of a pattern, $T$, to which the Kesten Pattern Lemma 2.2 applies:

Lemma 2.2. (Kesten Pattern Lemma) If there is a self-avoiding walk on which a pattern, e.g. $T$, occurs three times, then the number $w_{n}(\neg T)$ of $n$-step selfavoiding walks on which $T$ does not appear satisfies

$$
\lim \sup _{n \rightarrow \infty} n^{-1} \log w_{n}(\neg T)=\lambda(\neg T)<\kappa .
$$

To complete the proof of Theorem ?? one, therefore, requires the extension of the representation of a tight trefoil knot by a Kesten Pattern to any knot type. This is provided by Lemma 2.3:

Lemma 2.3. (Soteros, Sumners, \&3 Whittington 1992) [SSW92] Knots of any topological type are represented by a Kesten Pattern in the simple cubic lattice which forms a tight knot of that type.

Thus, if the number of self-avoiding walks not containing a knot of type $K$ is $w_{n}(\neg K)$

$$
w_{n}(\neg K) \leq w_{n}(\neg T) \leq e^{\lambda(\neg T) n+o(n)}<e^{\kappa n+o(n)} .
$$

Thus, the number of self-avoiding walks not containing a knot of type $K$ is an exponentially small fraction of the total number of self-avoiding walks.

This strategy also applies to the case of self-avoiding polygons because the removal of the last edge of a polygon can not create a pattern, implying that the number of n-edge based oriented self-avoiding polygons not containing the a knot of type $K$ is also an exponentially small fraction of the total number of self-avoiding polygons.

Immediate consequences of Theorem 2.1 include the following:

Corollary 2.4. All but exponentially few sufficiently long self-avoiding walks or polygons on the simple cubic lattice contain a knot of any selected topological type with positive density.

Corollary 2.5. The probability that a self-avoiding walk or polygon on the simple cubic lattice contains a knot (of any selected topological type) goes to one as the length goes to infinity.

Proof. The probability that an $n$-step random walk in the simple cubic lattice contains a knot given type, $\mathscr{P}_{K}(n)$, is bounded below by the probability that it contains the a tight knot of that type forming a Kesten Pattern, T, showing that

$$
1 \geq \mathscr{P}_{K}(n) \geq 1-\frac{w_{n}(\neg T)}{w_{n}} \geq 1-e^{(\lambda-\kappa) n+o(n)}
$$


and, therefore, that $\mathscr{P}_{K}(n) \rightarrow 1$ as $n \rightarrow \infty$.

As above, the strategy also applies to the case of the self-avoiding polygon because the deletion of the last edge of the polygon can not create a pattern, implying that the number of $n$-edge based oriented self-avoiding polygons not containing the $T$ configuration is not larger than the number of $(n-1)$-edge self-avoiding walks not containing $T$. As a consequence

$$
1 \geq \mathscr{P}_{K}(n) \geq 1-\frac{w_{n-1}(\neg T)}{p_{n}} \geq 1-e^{(\lambda-\kappa) n+o(n)}
$$

and, therefore, $\mathscr{P}_{K}(n) \rightarrow 1$ as $n \rightarrow \infty$.

Following the model provided by these arguments, we see that the key to the proof of the Slipknot Theorem 2.7 is the application of the Kesten Pattern Lemma to a slipknotted element, $S$, such as the one shown in Figure 3. Observe that this configuration can not be "reknotted" for the same reason that the Sumners-Whittington trefoil can not be unknotted, it is impossible for any other portion of the walk or polygon to penetrate the convex hull of the configuration. Thus we have a tight trefoil slipknot realized by a Kesten Pattern and can conclude that

$$
\lim \sup _{n \rightarrow \infty} n^{-1} \log w_{n}(\neg S)=\lambda(\neg S)<\kappa .
$$

Let $\mathscr{S}_{K}$ denote the set of random walks in simple cubic lattice containing a slipknot of type K. The argument would continue, as above, to show that all but exponentially few sufficiently long self-avoiding walks on the simple cubic lattice contain a slipknot of type $K$ and, therefore, the probability that a random walk contains an slipknot of type $K, \mathscr{P}_{\mathscr{S}_{K}}(n)$ satisfies

$$
1 \geq \mathscr{P}_{\mathscr{S}_{K}}(n) \geq 1-\frac{w_{n}\left(\neg \mathscr{S}_{K}\right)}{w_{n}} \geq 1-e^{\left(\lambda\left(\neg \mathscr{S}_{K}\right)-\kappa\right) n+o(n)}
$$

proving that $\mathscr{P}_{\mathscr{S}_{K}}(n) \rightarrow 1$ as $n \rightarrow \infty$.

Thus the generalization to slipknots and their associated ephemeral knots is achieved by the creation of lattice instances of tight slipknots to which the Lemma 2.2 also applies. This is the content of Lemma 2.6.

Lemma 2.6. Slipknots of any topological type, and their associated ephemeral knots, can be realized in the simple cubic lattice by tight Kesten Patterns.

A trefoil slipknot in the simple cubic lattice, inspired by the trefoil knot in Figure 2, is shown in Figure 3. Applying the MDS method to this trefoil slipknot, for a sample of size 10,000, one has a knotting spectrum consisting of $86.7 \%$ unknots and $13.3 \%$ trefoils. If one applies the MDS method to the ephemeral trefoil subsegment, for a sample of size of 10,000, one has a knotting spectrum consisting of $6.3 \%$ unknots, $92.76 \%$ trefoil knots, $0.44 \%$ figure-eight knots and, $0.47 \% 5_{1}$ knots thereby confirming its identification. While this example satisfies the requirements of the Kesten Pattern Lemma 2.2, Lemma 2.6 is required to complete the proof of the theorem in its full generality. 


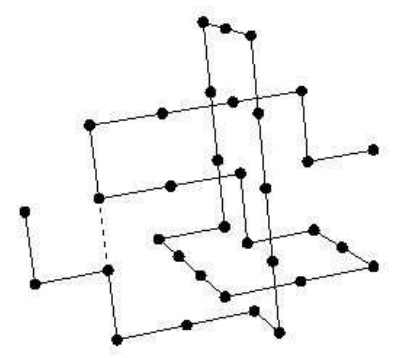

Figure 3: An trefoil slipknot with its trefoil ephemeral knot in the simple cubic lattice

Theorem 2.7. All except exponentially few sufficiently long self-avoiding walks or polygons on the simple cubic lattice contain ephemeral knots of any fixed topological type and their associated slipknots.

Immediate consequences of the theorem include the following:

Corollary 2.8. All but exponentially few sufficiently long self-avoiding walks or polygons on the simple cubic lattice contain an ephemeral knot and its associated slipknot of any selected topological type with positive density.

Corollary 2.9. The probability that a self-avoiding walk or polygon on the simple cubic lattice contains an ephemeral knot and its associated slipknot (of any selected topological type) goes to one as the length goes to infinity.

Corollary 2.10. (Whittington) The probability that a knotted self-avoiding walk or polygon in the simple cubic lattice contains a slipknot goes to one as the length goes to infinity.

The proof of Lemma 2.6 is inspired by Diao's the proof of the off-lattice theorem analogous to Theorem 2.1, Theorem 2.13, which we will also extend to slipknots in the next section.

Proof. (Lemma 2.6)

A strategy that one can employ in 3-space, as well as in the simple cubic lattice, is given by the knot doubling procedure that is illustrated in Figure 5. Here we show the construction for the classic figure-eight knot. One begins with the closure of a braid presentation of the figure-eight knot which is then "doubled" by adding a parallel strand. This doubled conformation is then modified by locally replacing a pair of corresponding parallel strands with the motif 

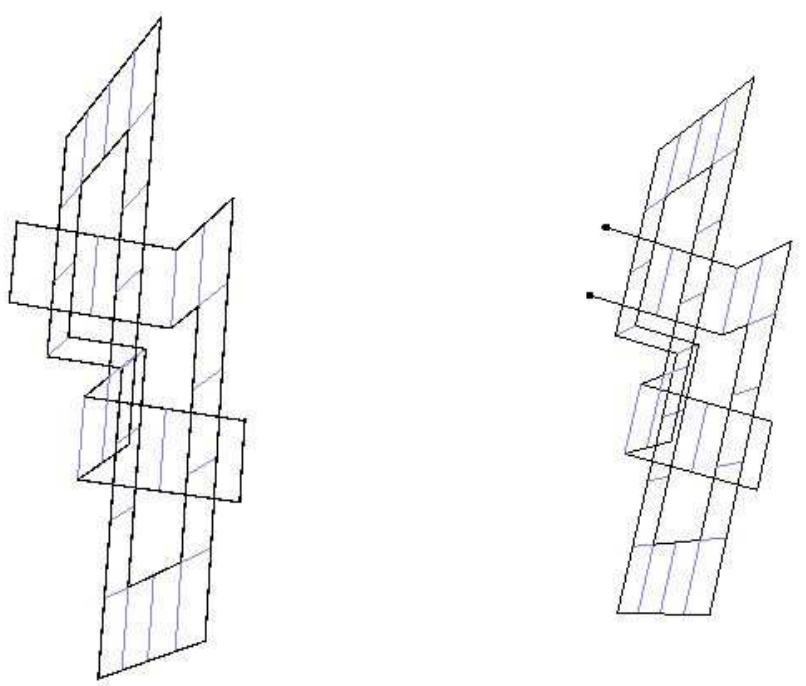

Figure 4: Creating a Kesten Pattern realizing a tight trefoil slipknot in the simple cubic lattice

shown in Figure 6. Beginning at the end vertex and following the strand to the second indicated vertex, one case traversed only the first half of the strand and has, in the sense above, a figure-of-eight knot. By continuing and thereby adding the second half of the strand to the first half, one "unties" the knot, in the informal sense of the term, and has constructed a figure-eight slipknot and its associated ephemeral knot.

We will employ an anologous construction in the simple cubic lattice. The first step is the creation of tight "ribbon" knot segment, for a tight trefoil ribbon, see Figure 4, of any topological type that will play a role analogous to the doubled braid described above. Their existence is guaranteed by the following Lemma $\left[\mathrm{JvROS}^{+}\right.$96]:

Lemma 2.11. (van Rensburg, Orlandini, Sumners, Tesi, and Whittington) Tight oriented ribbons with center line realizing any topological knot type can be realized in the simple cubic lattice by a Kesten Pattern.

The existence of a slip knot of this topological type follows the observation that removal of a bounding edge at the end of the ribbon creates a slipknot whose ephemeral knot it represented by following the ribbon "halfway" along the resulting path, see Figure 4.

Proof. (Theorem 2.7) 
The proof of Theorem 2.7 follows the argument of the proof of Theorem 2.1 with tight knots replaced by tight slipknots, i.e $\lambda(\neg T)$ is replaced in the proof by $\lambda(\neg S)$.

Proof. (Corrolary 2.10)

We will outline the proof in the case of self-avoiding walks in the simple cubic lattice, noting that the proof for polygons proceeds analogously. Recall that $w_{n}$ is the number of self-avoiding walks in the simple cubic lattice starting at the origin $\{0,0,0\}$, let $w^{K}{ }_{n}$ be the number of knotted walks, and let $w^{O}{ }_{n}$ be the number of unknotted walks. Let $w_{n}(\neg S)$ be the number of walks not containing a slipknot, let $w^{K}{ }_{n}(\neg S)$ be the number of knotted walks not containing a slipknot, and let $w^{O}{ }_{n}(\neg S)$ be the number of unknotted walks not containing a slipknot.

We recall that, $w_{n}=e^{n \kappa+o(n)}, w^{K}{ }_{n}=e^{n \kappa+o(n)}$, and $w^{O}{ }_{n}=e^{n \kappa} \kappa_{0}+o(n)$, with $\kappa_{0}<\kappa$. In addition, we know that

$$
w_{n}(\neg S)=e^{n \lambda(\neg S)+o(n)} \text { with } \lambda(\neg S)<\kappa .
$$

Since, $w^{O}{ }_{n}(\neg S) \leq w^{O}{ }_{n}=e^{n \kappa_{0}+o(n)}$ and $w_{n}(\neg S)=w^{K}{ }_{n}(\neg S)+w^{O}{ }_{n}(\neg S)$ we see that $w^{K}{ }_{n}(\neg S)=w_{n}(\neg S)-w^{O}{ }_{n}(\neg S) \leq w_{n}(\neg S)=e^{n} \lambda(\neg S)+o(n)$. As a consequence, we see that

$$
\lim \sup _{n \rightarrow \infty} n^{-1} \log w^{K}{ }_{n}(\neg S) \leq \lambda(\neg S)<\kappa=\lim \sup _{n \rightarrow \infty} n^{-1} \log w^{K}{ }_{n} .
$$

Therefore knotted walks not containing a slipknot are exponentially rare.

The prevous discussion of slipknots and their associated ephemeral knots suggests an observation as well as raising a number of questions. First, just as is the case with tight prime knots, one may form the connected sum of tight slipknots. As both are "tight" and can be realized by Kesten Patterns, the probability that a self-avoiding random walk or polygons in the simple cubic lattice contains this connected sum goes to one as the length goes to infinity. We are not, however, able to prove the following;

Conjecture 1. All but exponentially few sufficiently long unknotted self-avoiding walks or polygons in the simple cubic lattice contain a slipknot of any fixed topological type.

Can one distinguish between the knots and slipknots? We conjecture that their growth rates are the same, i.e:

Conjecture 2. In the simple cubic lattice, $\lambda(\neg K)=\lambda(\neg S)$. 


\subsection{Knots, Slipknots, and Ephemeral Knots in the 3-space}

Results paralleling those we have just described for the simple cubic lattice are also true in 3-dimensional Euclidean space. The model for the proof is the following theorem of Yuanan Diao [Dia95], itself a generalization of the Gaussian random knot proof [DPS94]:

Theorem 2.12. (Diao [Dia95]) All except exponentially few sufficiently long self-avoiding random walks and polygons in 3-space contain knots of any fixed topological type.

Specificially, we will prove:

Theorem 2.13. All except exponentially few sufficiently long self-avoiding random walks and polygons in 3-space contain slipknots of any fixed topological type and their associated ephemeral knots.

One then has, as above, the corollaries:

Corollary 2.14. All but exponentially few sufficiently long self-avoiding polygons and random walks in 3-space contain an ephemeral knot of any fixed topological type with positive density.

Corollary 2.15. The probability that self-avoiding polygon or random walk in 3space contains an ephemeral knot of any fixed topological type and its associated slipknot goes to one as the length goes to infinity.

Corollary 2.16. (Whittington) The probability that a knotted self-avoiding walk or polygon in the 3-space contains a slipknot goes to one as the length goes to infinity.

and the conjectures;

Conjecture 3. All but exponentially few sufficiently long unknotted self-avoiding walks or polygons in 3-space contain a slipknot of any fixed topological type.

Conjecture 4. In the 3-space, $\lambda(\neg K)=\lambda(\neg S)$.

We will briefly outline the proof of Diao's Theorem 2.12 and the modifications necessary to extend it to a proof of Theorem 2.13. First, every knot type, $K$, can be represented as a braid of $k$ strands and $m$ crossings showing, Diao argues, that it can be represented as an equilateral polygon of no more than $n=k(2 m+4)$ edges. Similarly, its double, see Figure 5 can be represented by

a braid of $2 k$ strands and $4 m$ crossings thereby implying that the double can be represented as an equilateral polygon ofno more than $n=2 k(8 m+4)$ edges. To complete the slipknot construction, we add an additional four edges, as shown in Figure 6, for a total of $n=2 k(8 m+4)+4$ edges.

Second, following Diao, assume that one has a cylinder of radius $r$ containing our modified double of the knot $K$, we estimate the probability that an equilateral conformation realizing this slipknot can be formed in the cylinder. 

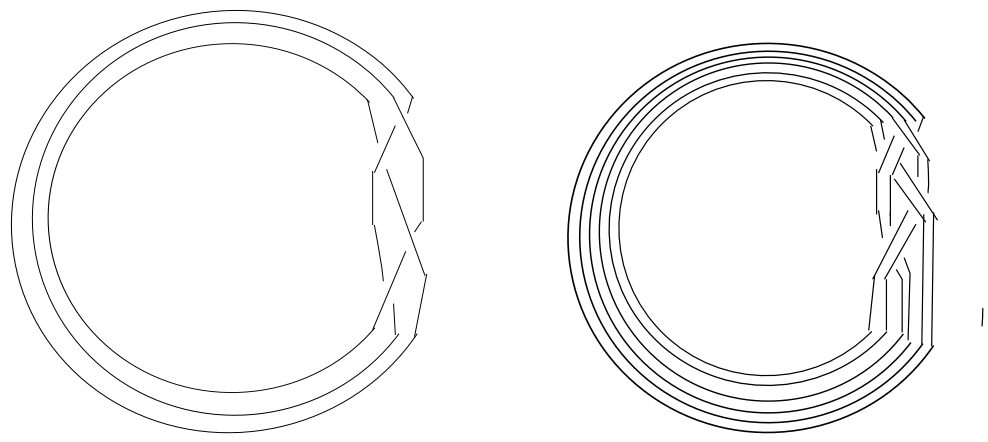

Figure 5: Doubling a braid representative of a knot
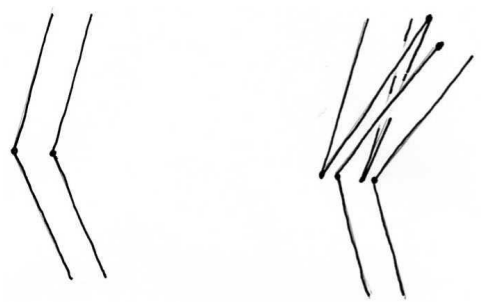

Figure 6: Creating an equilateral double from a braid representation

Diao's argument shows that there is a constant, $c^{\prime}>0$ so that if the vertices are perturbed by no more than $c^{\prime} r$, the type of the configuration is unchanged [MPR08]. The probability of this slipknot in the cylinder is at least $\mathrm{cr}^{2 n-3}$ for some constant $c>0$. The remainder of the proof follows as in [Dia95] and [DPS94].

\section{Conclusions}

King et. al. [KYY07] report the the identification of a slipknot deeply embedded in the structure of Escherichia coli alkaline phosphatase (PDB code 1ALK [KW91]). They find that the smallest segment of the protein that is knotted is between residues 51 and 371, forming a right-handed trefoil. Approximately 50 residues precede the segment on the $\mathrm{N}$-terminal side and, on the $\mathrm{C}$-terminal end, residues 371 to 419 form a loop that eventually "unknots" the trefoil. They found three other distinct protein folds, with PDB codes 1P6X, 2NWL, and 2 A65, having deeply embedded ephemeral knots as well as an additional candidate, 2J85, which was not as deeply embedded as the others. Inspired by the observation of these slipknots, we have proved that such structures occur in random walks and random equilateral polygons, in both the simple cubic lattice and in 3-space with asymptotic probability one. We have shown that any ephemeral knot type can occur and, furthermore, that these structures occur in 
knotted random walks and polygons with asymptotic probability one. We conjecture that the exponential growth rate of slipknots in knotted random walks and polygons is also the case for unknotted randomw walks and polygons. In addition, while one suspects that there should be a measurable difference between the growth rates for the occurrence of knots and of slipknots, we conjecture that the exponential factors associated with the growth of each are, in fact, equal.

We know that the presence of knots in a macromolecule has measurable consequences for its spatial and therefore physical and biological properties. What influence does the presence of slipknots have on these properties? Surely, we argue, the presence of the ephemeral knot that is reinforced by its becoming unknotted in a larger portion of the macromolecule, the entire slipknot, must have an even great consequence when they occur.

\section{Acknowlegments}

Thanks to Eric Rawdon, De Witt Sumners, Andrzej Stasiak, and Stuart Whittington for their interest and advice and to the Centre de Mathematiques et d'Iformatique (Marseille, France) for their hospitality.

\section{References}

[AET04] Greg Aloupis, Günter Ewald, and Godfried Toussaint. More classes of stuck unknotted hexagons. Beiträge Algebra Geom., 45(2):429-434, 2004.

[Cal98] J. A. Calvo. Geometric knot theory: the classification of spatial polygons with a small number of edges. $\mathrm{PhD}$ thesis, University of California, Santa Barbara, 1998.

[Cal01a] Jorge Alberto Calvo. The embedding space of hexagonal knots. Topology Appl., 112(2):137-174, 2001.

[Cal01b] Jorge Alberto Calvo. Geometric knot spaces and polygonal isotopy. J. Knot Theory Ramifications, 10(2):245-267, 2001. Knots in Hellas '98, Vol. 2 (Delphi).

[CJ98] Jason Cantarella and Heather Johnston. Nontrivial embeddings of polygonal intervals and unknots in 3-space. J. Knot Theory Ramifications, 7(8):1027-1039, 1998.

[CM98] J. A. Calvo and K. C. Millett. Minimal edge piecewise linear knots. In Ideal knots, pages 107-128. World Sci. Publishing, Singapore, 1998.

$\left[\mathrm{DDM}^{+} 03\right] \quad$ Akos Dobay, Jacques Dubochet, Kenneth Millett, PierreEdouard Sottas, and Andrzej Stasiak. Scaling behavior of ran- 
dom knots. Proc. Natl. Acad. Sci. USA, 100(10):5611-5615, 2003.

[Del62] M. Delbruck. Mathematical problems in the biological sciences. In Proc. Symp. Appl Math, volume 14, page 55, Providence, RI, 1962. American Mathematical Society.

[Deu99] J. M. Deutsch. Equilibrium size of large ring molecules. Phys. Rev. E, 59(3):2539-2541, 1999.

[Dia93] Yuanan Diao. Minimal knotted polygons on the cubic lattice. J. Knot Theory Ramifications, 2(4):413-425, 1993.

[Dia95] Yuanan Diao. The knotting of equilateral polygons in $\mathbf{R}^{3}$. J. Knot Theory Ramifications, 4(2):189-196, 1995.

[DPS94] Yuanan Diao, Nicholas Pippenger, and De Witt Sumners. On random knots. J. Knot Theory Ramifications, 3(3):419-429, 1994. Random knotting and linking (Vancouver, BC, 1993).

[Edw67] S. F. Edwards. Statistical mechanics with topological constraints: I. Proc. Phys. Soc, 91:513-519, 1967.

[FW61] H. L. Frisch and E. Wasserman. Chemical topology. Journal of the American Chemical Society, 83(18):3789-3795, 1961.

[Gro00] Alexander Yu. Grosberg. Critical exponents for random knots. Phys. Rev. Lett., 85(18):3858-3861, 2000.

[Ham61] J. M. Hammersley. The number of polygons on a lattice. Proc. Cambridge Philos. Soc., 57:516-523, 1961.

[HM54] J. M. Hammersley and K. W. Morton. Poor man's monte carlo. j. Roy. Statist. Soc. Ser. B, 16:23-38, 1954.

$\left[\mathrm{JvROS}^{+} 96\right] \quad$ E. J. Janse van Rensburg, E. Orlandini, D. W. Sumners, C. Tesi, and S. G. Whittington. Entanglement complexity of lattice ribbons. J. Stat. Phys., 85:103-130, 1996.

[JvRSWW92] E. J. Janse van Rensburg, D. A. W. Sumners, E. Wasserman, and S. G. Whittington. Entanglement complexity of selfavoiding walks. J. Phys. A, 25(24):6557-6566, 1992.

[Kes63] H. Kesten. On the number of self-avoiding walks. J. Mathematical Phys., 4:960 - 969, 1963.

$\left[\mathrm{KOV}^{+} 00\right] \quad$ V. Katritch, W. K. Olson, A. Vologodskii, J. Dubochet, and A. Stasiak. Tightness of random knotting. Physical Review E, 61:5545-5549, 2000. 
[KW91] E.E. Kim and H. W. Wyckoff. Reaction mechanism of alkaline phosphate based on crystal structures. two-metal ion catalysis. J. Mol. Biol., 218:449-464, 1991.

[KYY07] N. P. King, E. O. Yeates, and T. O. Yeates. Identification of rare slipknots in proteins and their implications for stability and folding. Journal of Molecular Biology, 373(1):153-166, 2007.

[Man94] M. Mansfield. Are there knots in proteins? Structural Biology, 1:213-214, 1994.

[MDS05] Kenneth Millett, Akos Dobay, and Andrzej Stasiak. Linear random knots and their scaling behavior. Macromolecules, 38(2):601-606, 2005.

[MOSZ05] B. Marcone, E. Orlandini, A. L. Stella, and F. Zonta. What is the length of a knot in a polymer? J. Phys. A, 38(1):L15-L21, 2005 .

[MOSZ07] B. Marcone, E. Orlandini, A. L. Stella, and F. Zonta. Size of knots in ring polymers. Phys. Rev. E, 75:041105-1-11, 2007.

[MPR08] Kenneth C. Millett, Michael Piatek, and Eric J. Rawdon. Polygonal knot space near ropelength-minimized knots. Journal of Knot Theory and its Ramifications, 17(5):601-631, 2008.

[MS05] K. C. Millett and B. M. Sheldon. Tying down open knots: a statistical method for identifying open knots with applications to proteins, volume 36 of Ser. Knots Everything, pages 203-217. World Sci. Publ., Singapore, 2005.

[OTJvRW98a] E. Orlandini, M. C. Tesi, E. J. Janse van Rensburg, and S. G. Whittington. Asymptotics of knotted lattice polygons. J. Phys. A, 31(28):5953-5967, 1998.

[OTJvRW98b] E. Orlandini, M. C. Tesi, E. J. Janse van Rensburg, and S. G. Whittington. Asymptotics of knotted lattice polygons. J. Phys. A, 31(28):5953-5967, 1998.

[Pip89] Nicholas Pippenger. Knots in random walks. Discrete Appl. Math., 25(3):273-278, 1989.

[SSW92] C. E. Soteros, D. W. Sumners, and S. G. Whittington. Entanglement complexity of graphs in $\mathbf{Z}^{3}$. Math. Proc. Cambridge Philos. Soc., 111(1):75-91, 1992.

[SW88] D. W. Sumners and S. G. Whittington. Knots in self-avoiding walks. J. Phys. A, 21(7):1689-1694, 1988.

[Tay00] W. Taylor. A deeply knotted protein structure and how it might fold. Nature, 406:916-919, 2000. 
[Tou01] Godfried Toussaint. A new class of stuck unknots in $\mathrm{Pol}_{6}$. Beiträge Algebra Geom., 42(2):301-306, 2001.

[VKK05] P. Virnau, Y. Kantor, and M. Kardar. Knots in globule and coil phases of a model polyethylene. J. Am. Chem. Soc., 127:1510215106, 2005.

[VMK06] P. Virnau, L. Mirny, and M. Kardar. Intricate knots in proteins: Function and evolution. PLOS Comp. Bio., 2:e122, 2006.

[WC86] S. A. Wasserman and N. R. Cozzarelli. Biochemical topology: applications to dna recombination and replication. Science, 232:951-960, 1986 .

[YNK08] T. O. Yeates, S. T. Norcross, and N. P. King. Knotted and topologically complex proteins as models for studying folding and stability. Current Opinion in Chemical Biology, 11:595-603, 2008. 